\title{
Early joint degeneration and antagonism between growth factors and reactive oxygen species. Is non-surgical management possible?
}

\author{
ANDREA FABIO MANUNTA ${ }^{1}$, PIETRO ZEDDE ${ }^{2}$, SEBASTIANO CUDONI ${ }^{2}$, \\ GIANFILIPPO CAGGIARI ${ }^{1}$, GIANFRANCO PINTUS ${ }^{3}$ \\ ${ }^{1}$ Orthopaedic Department, University of Sassari, Italy \\ 2 Orthopaedic and Traumatology Unit, Nuoro Hospital, Italy \\ ${ }^{3}$ Department of Biomedical Sciences, University of Sassari, Italy
}

\begin{abstract}
Purpose: in pathological conditions such as osteoarthritis (OA), overproduction of reactive oxygen species (ROS) may overwhelm the antioxidant defenses of chondrocytes, thus promoting oxidative stress and cell death. It can be hypothesized that increasing the antioxidant machinery of chondrocytes may prevent the age-associated progression of this disease. Growth factors (GFs) play an important role in promoting both the resolution of inflammatory processes and tissue repair. In view of these considerations, we set out to investigate the protective effect, against $\mathrm{H}_{2} \mathrm{O}_{2}$-induced oxidative cell death, potentially exerted by fluid drained from the joint postoperatively.

Methods: the present study was conducted in 20 patients diagnosed with bilateral knee osteoarthritis and treated, between January 2013 and June 2014, with prosthetic knee implantation on the side more affected by the arthritic process, together with intraoperative placement of a closed-circuit drainage aspiration system. As a result, 20 different serum samples were collected from the drained articular fluid, prepared using two different methodologies. In addition, forty blood serum samples were obtained and prepared: 20 from the surgically treated patients and 20 from healthy controls. The present work was undertaken to investigate the potential protective effect of
\end{abstract}

\section{Corresponding Author:}

Pietro Zedde, MD

Orthopaedic and Traumatology Unit, Nuoro Hospital

Viale San Pietro 43/B, 07100 Sassari, Italy

E-mail: pietrozedde@tiscali.it sera obtained from articular fluid drainage against hydrogen peroxide-induced oxidative stress in cultured human chondrocytes.

Results: exposure of chondrocytes to hydrogen peroxide elicited a dose-dependent increase in oxidative stress and chondrocyte cell death, phenomena that were significantly counteracted by the pre-treatment of cell cultures with sera from articular fluid drainage. Conclusions: oxidatively stressed chondrocytes treated with sera obtained from articular fluid drainage lived longer than those treated with blood serum samples and longer than untreated ones.

Clinical Relevance: synovial fluids are usually discarded once the drainage reservoir is full; instead they could benefit the patients from whom they are collected, as they are rich in growth factors and they may act as antagonists of ROS effects. Accordingly, they could be used to treat chondropathies, early OA, and mild OA located in other sites.

Keywords: reactive oxygen species, chondrocytes, growth factors, osteoarthritis, knee.

\section{Introduction}

Osteoarthritis $(\mathrm{OA})$ is a disease with a multifactorial etiology that causes loss of joint functionality due to processes that, in the early stages, involve the joint cartilage $(1,2)$. Both local and systemic factors can account for the development of OA. Different studies in the literature $(3,4)$ describe $\mathrm{OA}$ as the result of altered chondrocyte metabolism caused by activation of catabolic pathways, followed by release of pro-inflamma- 
tory cytokines, such as IL-1 $\beta$ and TNF $\alpha$. These factors are strongly involved in the activation of cellular and biochemical cartilage degeneration processes, such as the production of free radicals and chemokines, and the activation of several metalloprotease enzymes. Reactive oxygen species (ROS) are defined as chemical components containing oxygen with reactive chemical properties and they include free radicals such as superoxide $\left(\mathrm{O}_{2}\right)$ and the hydroxyl radical $(\mathrm{OH}-)$, as well as non-radical molecules such as hydrogen peroxide. ROS are constantly produced by a wide range of cellular processes and especially by oxidative phosphorylation. While low ROS levels are involved in the modulation of physiological cellular processes such as cell proliferation and differentiation, higher levels can affect the aforementioned cellular phenomena by causing apoptotic or necrotic cell death $(5,6)$. Human joint chondrocytes actively produce $\mathrm{ROS}$, such as $\mathrm{NO}, \mathrm{O}_{2^{-}}, \mathrm{HO}$, and $\mathrm{H}_{2} \mathrm{O}_{2}$, which are able to induce apoptosis in the chondrocytes themselves (7-11). Experimental studies based on joint chondrocytes have demonstrated that apoptotic cell death can be seen both in OA and in rheumatoid arthritis. In view of the ability of ROS to induce oxidative cell death, it can be hypothesized that increasing the cellular antioxidant machinery of chondrocytes may be a useful approach for preventing the age-associated progression of OA.

The evolution of OA leads to progressive joint deterioration and ultimately to the need, in more advanced phases, for patients to undergo prosthetic joint implant surgery; this is currently the only solution available to fully resolve the pain symptoms and achieve joint recovery. To facilitate the recovery, a drainage aspiration system is placed intraoperatively to prevent blood from collecting in the joint as a result of postsurgical bleeding. This is normally removed within 24-48 hours. Several studies have identified growth factors (GFs), and specifically TGF- $\beta$, as important elements promoting both the resolution of the inflammatory process and tissue repair (12-14). Using normal human chondrocytes as a study model, the present work was undertaken to analyze the protective effect, against $\mathrm{H}_{2} \mathrm{O}_{2}{ }^{-}$ induced oxidative cell death, potentially exerted by fluid drained from the joint postoperatively. The hypothesis of the study was that GFs potentially present in this fluid may exert a protective effect against oxidative damage of human joint chondrocytes.

\section{Methods}

\section{Participants and intervention}

The present study was conducted in 20 patients diagnosed with bilateral knee $\mathrm{OA}$ and treated, between January 2013 and June 2014, with a total knee replacement on the most affected side. The patients had a mean age of 70 years (range 58-74), and a male:female ratio of 2:3. Patients with coagulation problems, diabetes, liver diseases or malnutrition were excluded from the study. All the patients received anticoagulant prophylaxis based on low-molecular-weight heparin and antibiotic prophylaxis based on third-generation cephalosporins; they all underwent total knee arthroplasty using a posterior-stabilized implant (Nexgen LPS; Zimmer, Warsaw, IN, USA). The surgical technique was carried out through the placement of a tourniquet around the root of the extremity; a midline parapatellar arthrotomy and an intramedullary femoral and extramedullary tibial alignment were performed; the posterior cruciate ligament was sacrificed and antibiotic-impregnated cement was used to fix the tibial component. The surgery lasted an average of 85 minutes (range 70-100 minutes).

\section{Collection and preparation of serum samples}

After removal of the tourniquet and accurate hemostasis, a closed drainage aspiration system was placed. Tubular drainage systems in PVC or in silicone polyethylene were used; these were connected to a plastic foldable drainage pouch. The average quantity of fluid collected through drainage from the 20 patients was $261.5 \pm 56.1 \mathrm{ml}$ per patient. After 24 hours the drainage aspiration system was removed and the collected fluid was transferred to the lab, where it was treated: some was centrifuged in Falcon tubes and some in serumseparator tubes. The postoperative fluid treated in Falcon tubes was centrifuged at $2200 \mathrm{rpm}$ for 20 minutes at $37^{\circ} \mathrm{C}$. The postoperative fluid treated in serumseparator tubes was centrifuged at $2500 \mathrm{~g}$ for $10 \mathrm{minu}-$ tes at $10^{\circ} \mathrm{C}$. Once separation had taken place, only the superficial fluid was removed; this fluid was placed in five $1 \mathrm{ml}$ tubes and subsequently stored at $-80^{\circ} \mathrm{C}$.

In addition, 40 samples of blood serum were collected: 20 from the surgically-treated patients and 20 from healthy controls; blood was centrifuged at $10^{\circ} \mathrm{C}$ in serum-separator tubes at $2500 \mathrm{~g}$ for 10 minutes; as 
with the haemarthrosis fluid samples, the serum was then extracted and stored at $-80^{\circ} \mathrm{C}$. Afterwards the different samples were tested with different chondrocyte cultures.

During the postoperative period none of the 20 patients received whole blood units through transfusion.

\section{Chondrocyte cell cultures}

The human articular chondrocytes used in our study were isolated from human articular cartilage and provided by Innoprot (Biscay, Spain). Chondrocyte cultures are widely used as an in vitro model to study cartilage processes such as regeneration and repair, effects of cytokines and of GFs on cartilage, as well as the regulation of specific genes involved in cartilage physiopathology.

In order to study cell growth, cellular culture flasks were prepared, coated with poly-L-lysine $(2 \mu \mathrm{g} / \mathrm{cm} 2)$ (Sigma-Aldrich, Saint Louis, MO, USA). After confluence, cells were washed in phosphate buffered saline (PBS 1X; Sigma) and $8 \mathrm{ml}$ of PBS 1X were added and then $2 \mathrm{ml}$ of trypsin/EDTA solution in a T-75 flask. Cells were further incubated in $\mathrm{CO}_{2}$ at $37^{\circ} \mathrm{C}$ for $1-2$ minutes, or until they were completely separated, and checked with an inverted microscope. During incubation a conical tube for centrifugation was prepared with $5 \mathrm{ml}$ of fetal bovine serum (Lonza, Basel, Switzerland). Afterwards the trypsin/ EDTA solution was transferred from the flask to the $50 \mathrm{ml}$ centrifuge tube. The tube was centrifuged, (collected cells were suspended) at $1000 \mathrm{rpm}$ for 5 minutes and cells were re-suspended in the culture media. After that, cell counting was performed and cells were in a new poly-L-lysine-coated flask or disk at the recommended cell density.

\section{ROS determination and cell damage}

Determination of intracellular ROS levels was performed using 2', 7'-dichlorodihydrofluorescein diacetate (H2DCF-DA) (Molecular Probe, Eugene, OR, USA), a molecular probe specific for ROS. Cells were placed on a 96-well black plate at a concentration of $80,000 \mathrm{cells} / \mathrm{ml}$. Before treatment, the culture media had been replaced with PBS plus, containing H2DCF-DA $1 \mu \mathrm{M}$. The GENios plus microplate reader (Tecan, Männedorf, Switzerland) allowed fluorescence measurement using an excitation wavelength of $485 \mathrm{~nm}$ and an emission wavelength of $535 \mathrm{~nm}$ : the fluorescence variation induced by the treatment was kinetically measured every minute for 120 consecutive minutes (15). Cell damage was evaluated in 96-well plates using a colorimetric assay 3(4,5-dimethylthiazole-2-yl)-2,5-diphenyltetrazolium bromide (MT'T) (Promega, Madison, WI, USA) (16).

\section{Results}

First of all, we explored the ability of $\mathrm{H}_{2} \mathrm{O}_{2}$ to induce chondrocyte cell death due to oxidative stress. Exposure of chondrocytes to progressive concentrations of $\mathrm{H}_{2} \mathrm{O}_{2}$ resulted in loss of cell vitality proportional to the oxidant concentrations added. Cell vitality was expressed as percent of the control, using as control $(100 \%$ of vitality $=100 \%$ of absorbance $)$ cells that were not exposed to $\mathrm{H}_{2} \mathrm{O}_{2}$ (Fig. 1). In order to asses a potential protective effect of our serum samples, chondrocyte cultures were pre-treated with: i) haemarthrosis fluid containing serum, ii) haemarthrosis fluid without serum, and iii) blood serum, respectively, for six hours prior to exposure to $\mathrm{H}_{2} \mathrm{O}_{2}(300 \mu \mathrm{M})$. Vitality (absorbance reading) was verified after 24 hours' exposure to $\mathrm{H}_{2} \mathrm{O}_{2}$. The case samples demonstrated a reduction of vitality as compared to the controls, however,

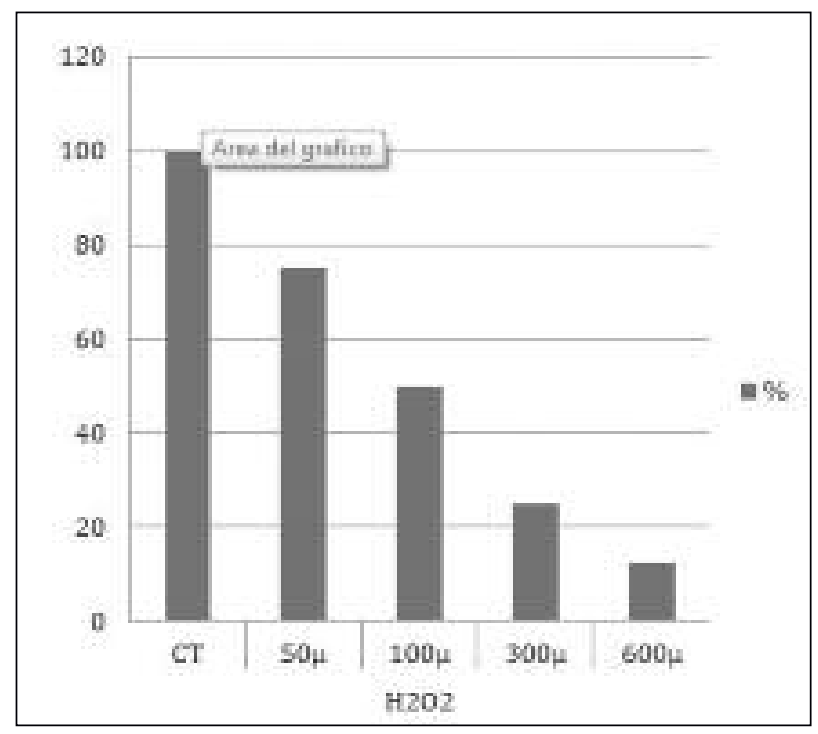

Fig. 1. The control cell samples not exposed to the treatment presented $100 \%$ vitality at 24 hours; conversely, a progressive vitality decrease was noted in the case samples. 


\section{oints}

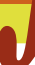

this reduction was minimal in culture media treated with haemarthrosis fluid without serum (Fig. 2).

We then investigated whether exposure time could be a factor involved in protection against $\mathrm{H}_{2} \mathrm{O}_{2}$. To this end, chondrocyte cultures were pre-treated with haemarthrosis fluid containing serum and without serum, and with blood serum, respectively, for 12 hours before exposure to $\mathrm{H}_{2} \mathrm{O}_{2}$. Vitality (absorbance reading) was again verified after 24 hours' exposure. Cell samples treated with blood serum showed a statistically significant reduction of vitality, while those treated with haemarthrosis fluid containing serum showed a not statistically significant reduction in vitality. Instead, the cells exposed to haemarthrosis fluid without serum had the same level of vitality as the controls (Fig. 3). In order to compare vitality between the different cell samples, the difference in vitality (absorbance) between controls and cases was evaluated. In the cultures pre-treated for six hours with haemarthrosis fluid without serum, cell death was significantly lower than in cultures pre-treated with haemarthrosis fluid containing serum and blood serum (Fig. 4).

Cell death in cultures pre-treated for 12 hours with haemarthrosis fluid without serum was almost identical to that found in controls, and cell death in the haemarthrosis fluid with serum pre-treated cultures was significantly reduced compared to that of the blood serum pre-treated cultures (Fig. 5).

\section{Discussion}

In the present study, we evaluated ROS-induced cell damage and the protective effect of GFs contained in haemarthrosis fluid with and without serum and in blood serum from a set of patients submitted to total knee arthroplasty. Our findings clearly demonstrate that increased oxidative stress is able to induce chondrocyte cell death, most likely by overwhelming the intracellular antioxidant systems of these cells. Since ROS levels, as well as ROS-associated oxidative stress, increase with aging (17), this aspect may be an important contributing factor to both the onset and the progression of $\mathrm{OA}$ in elderly.

Growth factors exert an important role in maintaining cartilage tissue homeostasis and the homeostasis of the joint structures as a whole (18-20). Thanks

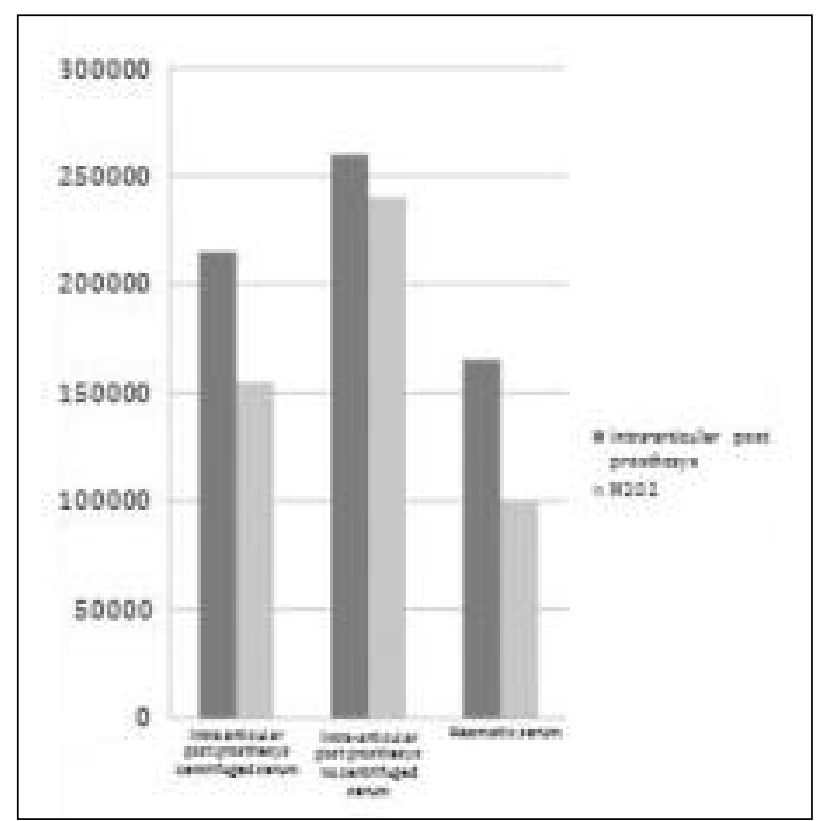

Fig. 2. Case cell samples demonstrated reduced absorbance compared with controls, however, this was minimal in culture treated with haemarthrosis fluid without serum.

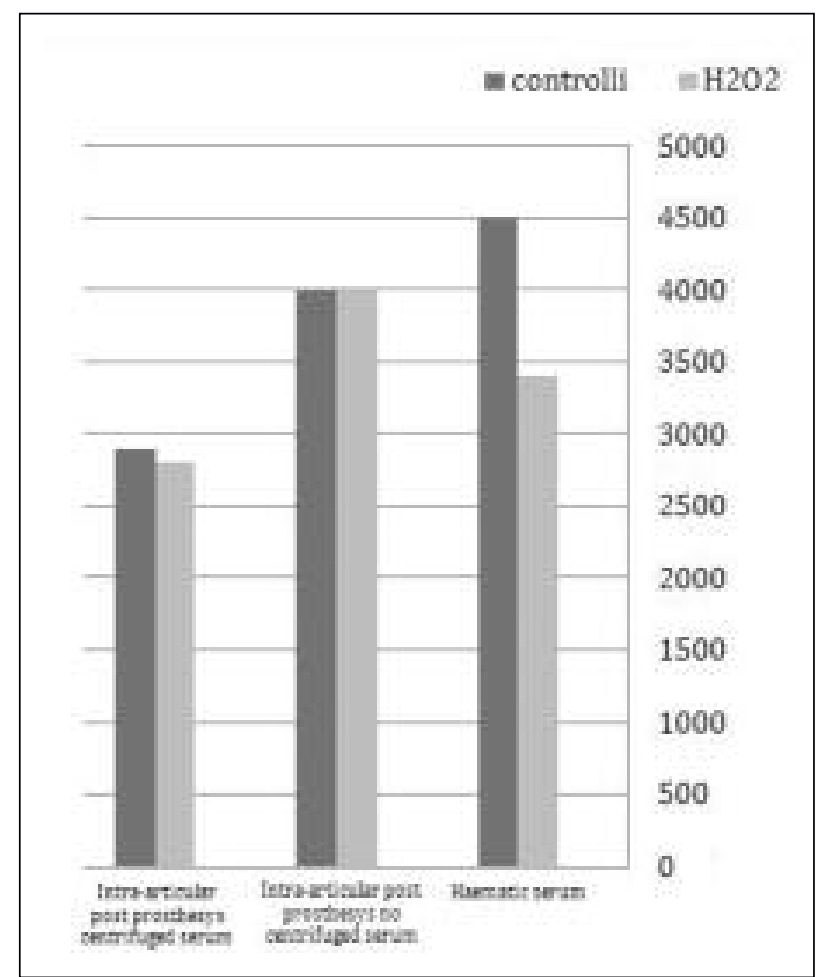

Fig. 3. Cell samples treated with blood serum showed a statistically significant reduction in absorbance. The absorbance of haemarthrosis fluid without serum had the same level as the controls. 


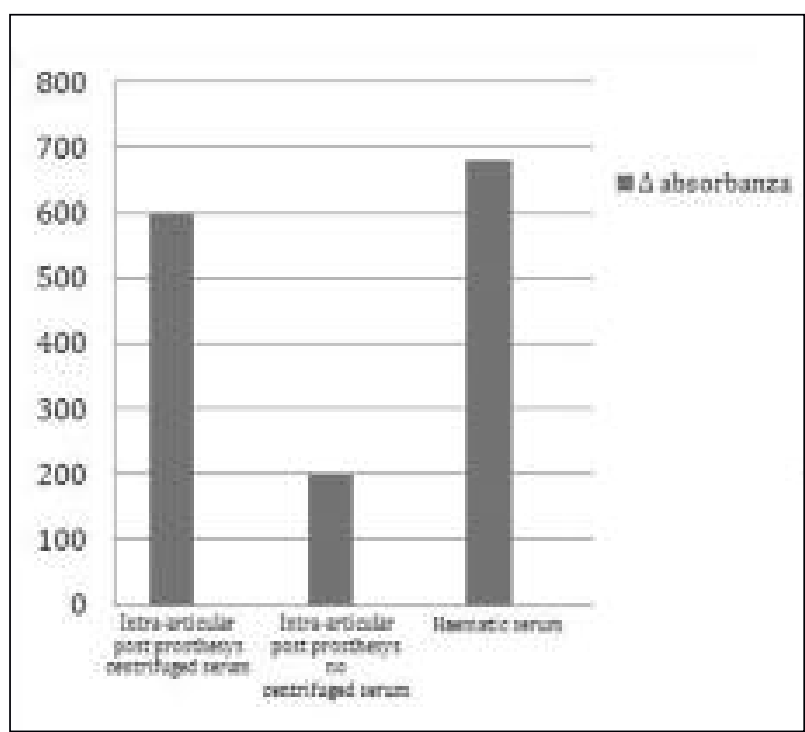

Fig. 4. In culture pre-treated for six hours with haemarthrosis fluid Fig. 4. In culture pre-treated for six hours with haemarthrosis fluid cultures pre-treated with haemarthrosis fluid containing serum and cultures pre-tr
blood serum.

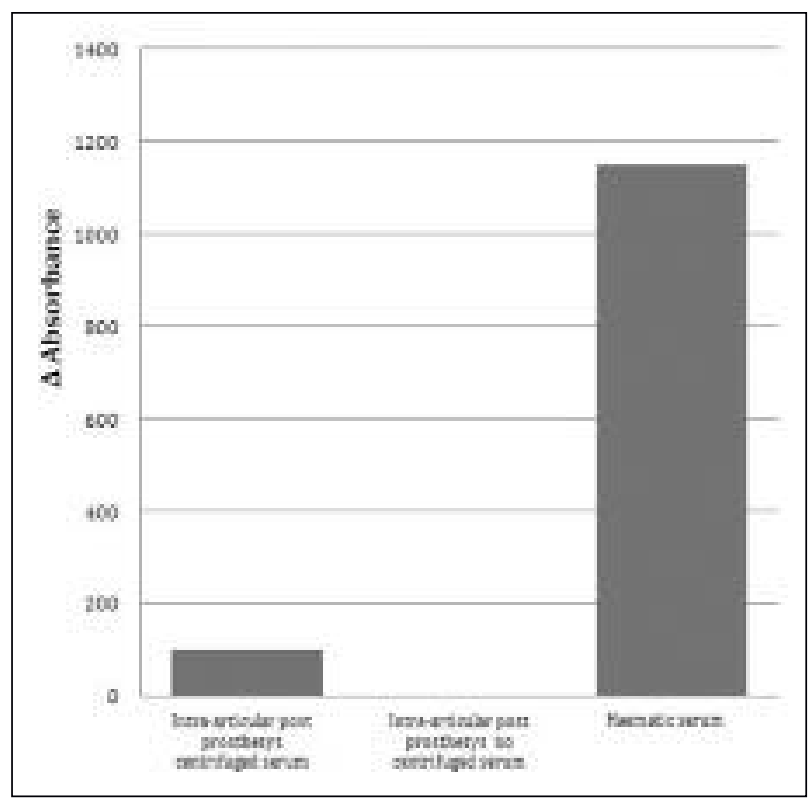

Fig. 5. Cell death in cultures pre-treated for 12 hours with haemarthrosis fluid without serum was almost identical that found in controls, and cell death in the haemarthrosis fluid with serum pre-treated cultures was significantly reduced compared to that of the blood serum pre-treated cultures.

to their coordinated secretion, which modulates a wide variety of physiological process of cells with a chondral phenotype, GFs help to stimulate differentiation of mesenchymal stem cells into the chondro- genic line; moreover, they promote matrix deposition, slow down matrix catabolism and reduce the suppressive effect on proteoglycan synthesis elicited by inflammatory mediators. In this context, plateletrich plasma (PRP), a concentrated source of autologous platelets, once applied into a specific surgical site, releases important GFs, including platelet-derived growth factor (PDGF), transforming growth factor- $\beta$ (TGF- $\beta$ ), fibroblast growth factor (FGF), insulin-like growth factor (IGF-1 and IGF-2) and vascular endothelial growth factor (VEGF), which promote physiological chondrocyte processes (21, 22). Akin to the therapeutic effects elicited by the PRP, our data clearly showed the protective effect mediated by the postoperative articular fluids, which, being collected from a site of surgery-induced inflammation, are potentially rich in GFs. This hypothesis is strongly supported by our data showing failure of the blood serum samples to promote chondrocyte protection, which indicated that GFs were present in high concentrations only in the postoperative articular fluids. Our results are consistent with the biological rationale for using PRP in degenerative joint pathologies, where the aim is, indeed, to achieve a high local concentration of GFs able to promote or restore the joint homeostasis (23-25). In fact, several in vitro studies, on animal and clinical models showed the efficacy of PRP to treat both chondral lesions and early degenerative joint pathologies (14, 26, 27). A noteworthy finding of our study was is that postoperative articular fluid without serum exerted a better protective effect than the fluid containing serum; this is probably due to different concentrations of GFs caused by the different procedures used to prepare the samples.

A limitation of this study is the fact that we may have used an endogenous pathophysiological source of oxidative stress (e.g. some inflammatory cytokines).

However, the molecular mechanisms of oxidativeinduced chondrocyte damage during the onset and progression of OA are far from clearly defined, therefore we believe that our model, at the moment, may be representative of a pathophysiologically relevant in vivo mechanism.

In conclusion, results obtained in the current study clearly showed the protective effect mediated by articular fluids containing serum and especially by those not containing serum. These fluids, usually discarded 


\section{oints}

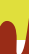

once the drainage reservoir is full, maybe instead used in the patients from whom they are collected to treat chondropathies, early OA or mild OA located in other sites.

\section{References}

1. Hochberg MC, Martel-Pelletier J, Monfort J, et al. Combined chondroitin sulfate and glucosamine for painful knee osteoarthritis: a multicentre, randomised, double-blind, noninferiority trial versus celecoxib. Ann Rheum Dis. 2015;14. doi: 10.1136/annrheumdis-2014-206792

2. van Saase JL, van Romunde LK, Cats A, et al. Epidemiology of osteoarthritis: Zoetermeer survey. Comparison of radiological osteoarthritis in a Dutch population with that in 10 other populations. Ann Rheum Dis. 1989;48:271-280.

3. Henrotin YE, Bruckner P, Pujol JP. The role of reactive oxygen species in homeostasis and degradation of cartilage. Osteoarthritis Cartilage. 2003;11:747-755.

4. Wang J, Verdonk P, Elewaut D, et al. Homeostasis of the extracellular matrix of normal and osteoarthritic human articular cartilage chondrocytes in vitro. Osteoarthritis Cartilage. 2003;11:801-809.

5. Valko M, Leibfritz D, Moncol J, et al. Free radicals and antioxidants in normal physiological functions and human disease. Int J Biochem Cell Biol. 2007;39:44-84.

6. Boonstra J, Post JA. Molecular events associated with reactive oxygen species and cell cycle progression in mammalian cells. Gene. 2004;4:337:1-13.

7. Stefanovic-Racic M, Morales TI, Taskiran D, et al. The role of nitric oxide in proteoglycan turnover by bovine articular cartilage organ cultures. J Immunol. 1996;156:1213-1220.

8. Hiran TS, Moulton PJ, Hancock JT. Detection of superoxide and NADPH oxidase in porcine articular chondrocytes. Free Radic Biol Med. 1997;23:736-743.

9. Tiku ML, Liesch JB, Robertson FM. Production of hydrogen peroxide by rabbit articular chondrocytes: enhancement by cytokines. J Immunol. 1990;145:690-696.

10. Del Carlo M Jr, Loeser RF. Nitric oxide-mediated chondrocyte cell death requires the generation of additional reactive oxygen species. Arthritis Rheum. 2002;46:394-403.

11. Bendinelli P, Matteucci E, Dogliotti G, et al. Molecular basis of anti-inflammatory action of platelet-rich plasma on human chondrocytes: mechanisms of NF-KB inhibition via HGF. J Cell Physiol. 2010;225:757-766.

12. Filardo G, Kon E, Buda R, et al. Platelet-rich plasma intraarticular knee injections for the treatment of degenerative cartilage lesions and osteoarthritis. Knee Surg Sports Traumatol Arthrosc. 2011;19:528-535.

13. Gobbi A, Bathan L. Biological approaches for cartilage repair. J Knee Surg. 2009;22:36-44.
14. Wang-Saegusa A, Cugat R, Ares O, et al. Infiltration of plasma rich in growth factors for osteoarthritis of the knee short-term effects on function and quality of life. Arch Orthop Trauma Surg. 2011;131:311-317.

15. Posadino AM, Cossu A, Giordo R, et al. Resveratrol alters human endothelial cells redox state and causes mitochondrial-dependent cell death. Food Chem Toxicol. 2015;78:1016.

16. Posadino AM, Cossu A, Giordo R, et al. Coumaric acid induces mitochondrial damage and oxidative-mediated cell death of human endothelial cells. Cardiovasc Toxicol. 2013;13:301306.

17. Dai DF, Chiao YA, Marcinek DJ, et al. Mitochondrial oxidative stress in aging and healthspan. Longev Healthspan. 2014: 3:6

18. Frazer A, Bunning RA, Thavarajah M, et al. Studies on type II collagen and aggrecan production in human articular chondrocytes in vitro and effects of transforming growth factorbeta and interleukin-1beta. Osteoarthritis Cartilage. 1994;2: 235-245.

19. Pujol JP, Chadjichristos C, Legendre F, et al. Interleukin-1 and transforming growth factor-beta 1 as crucial factors in osteorthritic cartilage metabolism. Connect Tissue Res. 2008; 49:293-297.

20. Schmidt MB, Chen EH, Lynch SE. A review of the effects of insulin-like growth factor and platelet derived growth factor on in vivo cartilage healing and repair. Osteoarthritis Cartilage. 2006;14:403-412.

21. Akeda K, An HS, Okuma M, Attawia M, et al. Platelet-rich plasma stimulates porcine articular chondrocyte proliferation and matrix biosynthesis. Osteoarthritis Cartilage. 2006;14: 1272-1280.

22. Wu W, Chen F, Liu Y, et al. Autologous injectable tissue-engineered cartilage by using platelet-rich plasma: experimental study in a rabbit model. J Oral Maxillofac Surg. 2007;65:19511957.

23. Anitua E, Andia I, Ardanza B, et al. Autologous platelets as a source of proteins for healing and tissue regeneretion. Thromb Haemost. 2004;91:4-15.

24. Andad SX, Viles-Gonzales JF, Badimon JJ, et al. Membraneassociated CD40L and sCD40L in atherothrombotic disease. Thromb Haemost. 2003;90:377-384.

25. Saito M, Takahashi KA, Arai Y, et al. Intraarticular administration of platelet-rich plasma with biodegradable gelatin hydrogel microspheres prevents osteoarthritis progression in the rabbit knee. Clin Exp Rheumatol. 2009;27:201-207.

26. Sánchez M, Anitua E, Azofra J, et al. Intra-articular injection of an autolgous preparation rich in growth factors for the treatment of knee OA: a retrospective cohort study. Clin Exp Rheumatol. 2008;26:910-913.

27. Kon E, Buda R, Filardo G, et al. Platelet-rich plasma: intraarticular knee injections produced favorable results on degenerative cartilage lesions. Knee Surg Sports Traumatol Arthrosc. 2010;18:472-479. 